\title{
A Method for Computing the Kernel of $n$-Person Games ${ }^{1}$
}

\author{
By R. J. Aumann, B. Peleg and P. Rabinowitz
}

1. Introduction. During the last three years we have been witnessing the growth of a new theory for $n$-person characteristic-function games-the theory of bargaining sets. This theory tries to answer the following basic question: Given a partition of the players into coalitions, how should the payoff to each of these coalitions be divided among its members?

Various answers are given in [1], [4] and [3], where several bargaining sets are defined. The bargaining sets are sets of "stable" payoff distributions; roughly speaking, a payoff distribution is "stable" if the players in each coalition can effectively counter any threats by their coalition partners to obtain higher payoffs by leaving the old coalition in order to combine with outside players. This intuitive notion can be made precise in a number of different ways, leading to the various bargaining sets.

For several bargaining sets there are general existence theorems ([4], [11] and [3]); for other bargaining sets, general existence theorems cannot be proved [1, Section 7]. After the existence question had been settled, the problem of computing the bargaining sets arose. Since every bargaining set is given by a system of linear inequalities in the space of the payoffs [1, Theorem 2.1] there is no difficulty in principle, and the only problem is to find a practical method of computation. In this paper we give such a method for the kernel, a bargaining set that was defined and investigated by Davis and Maschler in [3], and has been the subject of considerable subsequent research $[9,12,13]$. The kernel is the easiest of the bargaining sets to compute.

The computational method described here is practical for games with 5 or fewer players, whose characteristic functions take small integer values. For such games, a computer program was written for the CDC-1604A at the Weizmann Institute of Science; all computations reported here were performed on that machine. The program was used to compute the kernels of all weighted majority games with 5 or fewer players, and all extreme zero-sum games with 5 players; the results of these computations are tabulated in this paper.

The kernel is defined in $\S 2$. In $\S 3$ we discuss the problems connected with computing the kernel, and describe our method. $\$ 4$ contains several estimates needed for the programming of the method. In $\S \S 5$ and 6 a detailed description of the program is given. The tables mentioned above are in $\$ \S 7$ and 8, together with descriptive material; in particular, in $\$ 7$ it is shown how the table in that section may be applied to certain games with more than 5 players.

It should be emphasized that the method used here is applicable for a class of

Received June 22, 1964.

1 The research described in this paper was partially supported by the U.S. Office of Naval Research, Logistics and Mathematical Statistics Branch, under Contract Number N625583586 . 
computational problems of which the bargaining set is typical, but that need have no relation with game theory. Specifically, if a set in euclidean space is defined by a (possibly large) number of linear inequalities connected (possibly in a very com. plicated way) by "or" and "and", and if it is desired to express the set as a union of convex polyhedra, then the method of this paper may be applicable if the dimension is sufficiently small and the coefficients of the inequalities are small integers.

2. Definitions. In this section we give the basic definitions that are used in the rest of the paper.

An $n$ person game is a set $N$ with $n$ members, together with a real function $v$, defined on the subsets of $N . v$ is the characteristic function of the game. The members of $N$ are called players, and will be denoted by the numbers $1, \cdots, n$. Subsets of $N$ are called coalitions. We assume that $v$ satisfies the following conditions: $v(\{i\})=0$ for $i=1, \cdots, n, v(\varnothing)=0$, and $v(S) \geqq 0$ for all $S \subset N$.

Let $(N, v)$ be an $n$-person game. A coalition structure is a partition of $N$. Intuitively, when $(N, v)$ is played, the players are partitioned according to a certain coalition structure, and each coalition $S$ in the partition divides its share $v(S)$ among its members. A payoff vector is an $n$-tuple $\left\{x_{i}\right\}_{i \in N}$ of real numbers, ${ }^{2}$ one number for each $i \in N$. If each player refuses to receive less than what he can get alone, namely zero, then a possible outcome of the game, which will be called an individually rational payoff configuration, is a pair $(x, B)$, where $B$ is a coalition structure, and $x$, the distribution of the payoffs, is a payoff vector that satisfies: $x_{i} \geqq 0$ for $i=1, \cdots, n$, and $\sum_{B} x_{i}=v(B)$ for all $B \in \mathbb{B}$.

Let $(x, B)$ be an individually rational payoff configuration. For each coalition $S$, set

$$
e(S, x)=v(S)-\sum_{S} x_{k} .
$$

Now let $i, j \in B \in \mathbb{B}$ and $i \neq j$; set

$$
T_{i j}=\{S: S \subset N, i \in S \text { and } j \notin S\},
$$

and

$$
s_{i j}(x)=\max \left\{e(S, x): S \in T_{i j}\right\} .
$$

We say that $i$ outweighs $j$ if $x_{j}>0$ and $s_{i j}(x)>s_{j i}(x)$. The payoff vector $x$ is balanced if there exists no pair of players $h$ and $k$ in the same $B$ in $B$ such that $h$ outweighs $k$. The kernel is the set of all balanced payoff vectors. It depends, of course, on the coalition structure $B$.

Intuitively, when $i$ and $j$ are in the same coalition of $B$ and $s_{i j}(x)$ is non-negative, then it is the maximum amount with which $i$ can "manouevre" (i.e. take for himself or offer to his partners) in case he wants to set up a new coalition that will exclude $j$. When $s_{i j}(x)$ is negative it no longer has the above meaning, but we think it is still a measure of $i$ 's power to threaten $j$. Of course player $j$ is immune from threats if $x_{j}=0$, for he can get 0 without help from any other player. But if $x_{j}>0$, then $i$ outweighs $j$ if $i$ 's power to threaten $j$ exceeds $j$ 's power to threaten $i$.

${ }^{2}$ Formally, it is a function from $N$ to the real numbers. 
The payoff vector is balanced if no player outweighs another player in the same coalition.

The reader who wishes a more extensive intuitive and theoretical (rather than computational) discussion of the kernel concept, as well as a detailed discussion of the kernel of a particular game, is referred to [3]. Also, in [3] it is proved that the kernel is never void, i.e. if $(N, v)$ is an $n$-person game and $B$ is a coalition structure, then there always exists a payoff vector $x$ that is balanced [3, Theorem 5.4]. However, most of the methods of this paper do not depend on [3], and a reader not familiar with [3] should have no difficulty in understanding this paper.

3. Method of Computation. Let $(N, v)$ be an $n$-person game and let $B$ be a coalition structure. Denote by $X(B)$ the set of all payoff vectors $x$ such that $(x, B)$ is an individually rational payoff configuration. Explicitly,

$$
X(B)=\left\{x: x_{i} \geqq 0 \text { for } i=1, \cdots, n \text {, and } \sum_{B} x_{i}=v(B) \text { for } B \in Q\right\} .
$$

Our problem is to find those payoff vectors in $X(B)$ that belong to the kernel.

The computational method that we use is based on the fact that for each $B$, the kernel is a polyhedron that is not necessarily convex; that is, it is a finite union of convex polyhedra. To see this, note that a vector $x$ is in the kernel if and only if

$$
x \in X(B) \text { and for } i=1, \cdots, n, \quad x_{i}=0 \text { or } s_{i j}(x) \geqq s_{j i}(x)
$$

for all $j \in B-\{i\}$, where $B$ is that coalition of $B$ that contains $i$.

Now $s_{i j}(x)$ (and therefore also $s_{j i}(x)$ ) is the maximum of a finite number of linear functions of $x$, i.e. functions of the form $a \cdot x+c$, where $a$ is a vector and $c$ a scalar. Therefore (3.1) may be stated as a sentence $\delta$ built from weak linear inequalities, by means of iterated conjunctions and disjunctions (i.e. by using the connectives "and" and "or"). Now any sentence built in such a way from a number of "primitive statements" (in our case linear inequalities), no matter how complex its structure, is equivalent to an appropriately chosen disjunction of conjunctions of the primitive statements. Therefore there are linear functions $g_{p q}(x)$, where $p$ and $q$ run over finite index sets (say from 1 to $P$ and 1 to $Q$ respectively), such that (3.1) is equivalent to: There is a $p$ such that for all $q, g_{p q}(x) \geqq 0$.

Each of the $g_{p q}(x)$ appears in the sentence $\mathcal{S}$, and it may be verified that each one is therefore either of the form $\sum_{s} x_{i}-v(S)$ for some $S$, or of the form $\sum_{s} x_{i}-$ $\sum_{T} x_{i}-(v(S)-v(T))$ for some $S$ and $T$; in particular, the $g_{p q}$ have rational coefficients only, except possibly for the constant term. From the form (3.2) it follows immediately that the kernel is the union of $P$ convex polyhedra, each determined by $Q$ linear inequalities.

The process of reducing a complex sentence like (3.1) to the form (3.2) will be familiar to the reader from the Propositional Calculus; it consists essentially of repeated applications of the distributive law

$$
a \text { and }(b \text { or } c) \Leftrightarrow(a \text { and } b) \text { or }(a \text { and } c) .
$$

For fixed $n$ and $B$ it is of course possible explicitly to carry out this procedure, 
thereby obtaining numerical values for $P$ and $Q$ and listing all the $g_{p q}$ (with the values of $v$ entering as parameters). But our purpose in the foregoing was merely to show that the kernel is the union of finitely many convex polyhedra; this we have done, and for this purpose it is not necessary to exhibit $P, Q$, and the $g_{p q}$ explicitly.

We wish to "compute" the kernel; what does this mean? If the kernel consists of one point, we would like to know the coordinates of that point; if it is an interval we would like the end points of the interval; if it is the union of two intervals, we would like the end points of each of the intervals. As we have seen, the kernel is a finite union of convex polyhedra; the end result of a "computation" of the kernel should be a listing of these convex polyhedra, where each one of them is described by listing its extreme points (i.e. vertices). Theoretically, we know how to achieve a breakdown into convex polyhedra: by using the rules of the propositional calculus to change (3.1) to (3.2). Possibly this approach could be used on a practical, computational level as well. Essentially, (3.2) presents the kernel as the union of $P$ convex polyhdera, each one defined by a set of linear inequalities. A practical method for finding the vertices of a convex polyhedron defined by a set of inequalities is given by Balinski in [2]. Couldn't we find (3.2) explicitly, then use Balinski's method to calculate the vertices of each of the $P$ resulting convex polyhedra?

The answer is no, and the reason is that $P$ is very large-for $n=5$, probably on the order of $10^{4}$ or $10^{5}$. To apply Balinski's method to so many polyhedra would be prohibitive. The very large value for $P$ might lead the reader to think that it is really necessary to use many convex polyhedra to describe a kernel, but nothing could be further from the truth. The kernel is usually very simply described; indeed, it often consists of a single point. In the tables in $\$ \$ 7$ and 8 , there are only three instances in which the kernel is not itself convex, and in those cases it is the union of 2 convex polyhedra. The representation (3.2) is inefficient, because it holds for all games simultaneously; the characteristic function enters into the individual $g_{p q}$ only as a parameter, and affects neither $P$ nor $Q$. For a particular game, most of the $P$ convex polyhedra are empty, or coincide with each other. What is needed is some way of computing the kernel without first writing it explicitly in the form (3.2). We shall now outline our solution to this problem.

From now on it will be assumed that $(N, v)$ is integral, i.e. that the characteristic function $v$ takes integer values only. In theory, everything in the sequel applies to arbitrary integral games, and so by $S$-equivalence $[8$, p. 197] to arbitrary rational games. In practice, though, the method is useable only when the values of $v$ are small integers, probably 5 or 6 at most.

For the given game $(N, v)$ and coalition structure $B$, denote by $C_{1}, \cdots, C_{P}$ the convex polyhedra defined by (3.2); that is,

$$
C_{p}=\left\{x \in E^{n}: g_{p q}(x) \geqq 0 \text { for } q=1, \cdots, Q\right\} \text {. }
$$

A given $C_{p}$ may be empty, or it may coincide with others having different indices. The purpose of the computation is to provide a list of the nonempty $C_{p}$ 's, each one exactly once, and each one described by listing its extreme points. The straightforward way of doing this would seem to be first somehow to characterize the $C_{p}$ 's in question, then to determine the extreme points of each one. For the reasons explained above, this straightforward way is impractical. The approach we use is apparently "upside-down": first all the extreme points of all the $C_{p}$ are listed, and only afterwards are they sorted out. 
Let $x$ be one of the extreme points in question. $x$ is the unique solution of a system of $n$ linear equations, which have one of the following two forms: $\sum_{s} x_{i}=$ $v(S)$, or $\sum_{s} x_{i}-\sum_{r} x_{i}=v(S)-v(T)$. Since the game is integral, $x$ must be a rational point, and it has a denominator which does not exceed a bound $K_{n}$, which is not greater than the maximal value attained by a determinant of order $n$ whose entries are 1,0 , or -1 . Now, if for each rational point in $X(B)$ with denominator ${ }^{3} \leqq$ $K_{n}$, we determine whether or not that point is in the kernel (i.e. satisfies the inequalities (3.1)), we obtain a list of points that contains all the extreme points in question. Moreover, if with each point $x$ that we have found in this wey we list the values $e(S, x)$ for all $S \subset N$, then we shall be able to group the points according to the various $C_{p}$ to which they belong. It remains only to determine whether a given $x$ is actually extreme in at least one of the $C_{p}$; how this is done is described below.

Strictly speaking, the method just outlined can be exploited only to solve 4person games; when $n>4$, there are too many rational points with denominator $\leqq$ $K_{n}$. To overcome this difficulty, we use the following procedure: first, for a certain natural $K$ we examine all the rational points with denominator $K$, to find which of them are near the kernel, i.e. satisfy the inequalities (3.1) approximately (within $\epsilon$, say). This part of the procedure is called step $\mathrm{A}$. We then note that the kernel itself must be contained in a neighborhood of the set of points found in step A, the size of the neighborhood being determined by $\epsilon$. Now the extreme points that we are seeking must all be in this neighborhood, must be rational with denominator not exceeding $K_{n}$, and must satisfy the inequalities (3.1) - or equivalently, (3.2)precisely. Moreover, for each such extreme point $x$, the rank of those of the linear inequalities in (3.2) that are actually satisfied as equalities by $x$ must be $n$; indeed this is a necessary and sufficient condition for extremality. We therefore check the inequalities (3.1) for all points in $X(B)$ with denominators $\leqq K_{n}$, that are in the neighborhood determined by step A. Those points that are found to satisfy the inequalities are then examined for extremality by the rank method. This second (and last) part of the procedure is called step B.

To avoid round-off, integer arithmetic was used throughout the program.

4. Various Estimates. In this section we shall compute certain bounds needed for the programming of steps A and B for five person games.

We denote by $Q_{n}$ the maximal value attained by a determinant of order $n$ whose entries are 1,0 , or -1 . Hadamard's inequality yields $Q_{n} \leqq n^{n / 2}$, so $Q_{5} \leqq 5^{5 / 2} \approx 55.9$. In fact, $Q_{5}=48$. Actually we are interested not in $Q_{5}$ but in $K_{5}$.

LEMMA 4.1. $K_{5} \leqq 36$.

Proof. Let $A$ be a $5 \times 5$ regular matrix whose entries are 0,1 or $-1, b$ an integral vector and $x$ the solution of the system of equations $A x=b$. If all the zeros of $A$ are contained in at most two rows or two columns, then $|A| \equiv 0(\bmod 4)$, and each minor of order 4 is a multiple of 2 . So in this case the denominator of $x$ does not exceed $48 / 2=24$. Next, suppose that the zeros of $A$ are not in two rows or two columns. Clearly, it is sufficient to show that when there are exactly three zeros, ${ }^{4}$

${ }^{8}$ I.e., least common denominator of all the coordinates.

4 If there are four zeros, not contained in two rows or two columns, then Hadamard's inequality gives $|A| \leqq 36$. 
$|A| \leqq 36$. W.l.o.g. we may assume that $|A|$ has the following form:

$$
\left|\begin{array}{ccccc}
0 & a_{12} & a_{13} & a_{14} & 1 \\
a_{21} & 0 & a_{23} & a_{24} & 1 \\
a_{31} & a_{32} & 0 & a_{34} & 1 \\
a_{41} & a_{42} & a_{43} & a_{44} & 1 \\
1 & 1 & 1 & 1 & 1
\end{array}\right| .
$$

We now develop $|A|$ with respect to the last two rows, using Laplace's rule. It is not difficult to see that at most six of the $2 \times 2$ determinants that appear are nonzero, and that of the three $3 \times 3$ determinants with only one zero, only one can equal 4, and the other two are not greater than 2; the other determinants are not greater than 3 . Using these remarks we obtain $\left|K_{5}\right| \leqq 36$.

The exact value of $K_{5}$ is not known.

Let $(N, v)$ be an integral 5 -person game, and let $\&$ be a coalition structure. The number of rational points in $X(B)$ with denominator $K$ depends on the values $v(B)$ for $B \in B$. We shall now compute it in the following simple case: $B=\{N\}$ and $v(N)=1$. Denote by $Z(n, K)$ the number of integral points satisfying $x_{i} \geqq 0$, $i=1, \cdots, n$ and $\sum_{i=1}^{n} x_{i}=K$. Then

$$
Z(n, K)=\sum_{j=0}^{K} Z(n-1, j) .
$$

Using this relation we obtain

$$
Z(5, K)=\left(K^{4}+10 K^{3}+35 K^{2}+50 K+24\right) / 24 .
$$

The number of rational points in $X(B)$ with denominator not exceeding $K_{5}$ is bounded by $\sum_{k=1}^{36} Z(5, K)$, which is of the same order of magnitude as $\sum_{k=1}^{36} K^{4} / 4 ! \approx 36^{5} / 5$ !

The following lemma gives an estimate of the density of the rational points in $X(B)$ with given denominator.

Lemma 4.4. Let $K$ be a natural number. If $x \in X(B)$, then there is a rational point $y \in X(B)$ with denominator $K$, such that:

$$
\begin{gathered}
\text { if } x_{i}=0 \text { then } y_{i}=0, \quad \text { and } \\
\left|x_{i}-y_{i}\right| \leqq 4 /(5 K)=\delta(K), \text { for } i=1, \cdots, 5 .
\end{gathered}
$$

The proof, which is elementary, is omitted. We remark that the bound $4 /(5 K)$ cannot be improved.

We now want to estimate the rate of variation of the functions $s_{i j}(x)$. A convenient norm is $\|x\|=\max \left\{\left|x_{1}\right|, \cdots,\left|x_{5}\right|\right\}$.

Lemma 4.5. Let $i, j \in B \in \mathbb{B}, i \neq j$, and let $h_{i j}(x)=s_{i j}(x)-s_{j i}(x)$, for $x \in$ $X(B)$. If $x$ and $x^{\prime}$ are in $X(B)$, then $\left|h(x)-h\left(x^{\prime}\right)\right| \leqq 4\left\|x-x^{\prime}\right\|$.

Proof. Let $s_{i j}(x)=e(S, x), s_{i j}\left(x^{\prime}\right)=e\left(S^{\prime}, x^{\prime}\right), s_{j i}(x)=e(T, x)$ and $s_{j i}\left(x^{\prime}\right)=$ $e\left(T^{\prime}, x^{\prime}\right)$. We may assume that $h(x) \geqq h\left(x^{\prime}\right)$; then we have $\left|h(x)-h\left(x^{\prime}\right)\right|=$ $h(x)-h\left(x^{\prime}\right)=e(S, x)-e(T, x)-\left\{e\left(S^{\prime}, x^{\prime}\right)-e\left(T^{\prime}, x^{\prime}\right)\right\} \leqq e(S, x)-$ $e\left(S, x^{\prime}\right)+e\left(T^{\prime}, x^{\prime}\right)-e\left(T^{\prime}, x\right)=\sum_{s}\left(x_{k}{ }^{\prime}-x_{k}\right)+\sum_{r^{\prime}}\left(x_{k}-x_{k}{ }^{\prime}\right)$. If $S \cap T^{\prime} \neq \varnothing$ or $S \cup T^{\prime} \neq N$, then the number of the differences that are not cancelled does not exceed 4. So, in this case $\left|h(x)-h\left(x^{\prime}\right)\right| \leqq 4\left\|x-x^{\prime}\right\|:$ If $S \cap T^{\prime}=\varnothing$ and 
$S \cup T^{\prime}=N$, we have $\sum_{s}\left(x_{k}{ }^{\prime}-x_{k}\right)=\sum_{T^{\prime}}\left(x_{k}-x_{k}{ }^{\prime}\right)$. One of the sets, $S$ or $T^{\prime}$, does not contain more than two players; so also in this case $\left|h(x)-h\left(x^{\prime}\right)\right| \leqq$ $4\left\|x-x^{\prime}\right\|$. This completes the proof of the lemma.

The following definitions and lemma are the core of step A.

A subset $A$ of $X(B)$ is a $\delta$-kernel, if for each $x$ in the kernel there is a $y \in A$ such that $\|y-x\| \leqq \delta$. A point $x \in X(B)$ is $\epsilon$-balanced if it satisfies the following systems of inequalities:

$$
\text { for } i=1, \cdots, n \quad x_{i}=0, \quad \text { or } \quad s_{i j}(x) \geqq s_{j i}(x)-\epsilon
$$

for all $j \in B-\{i\}$, where $B$ is that coalition of $B$ that contains $i$.

Lemma 4.7. Let $K$ be a natural number. The set of rational points in $X^{\prime}(B)$ with denominator $K$ that are $3 / K$-balanced, is a 4/5-kernel.

Proof. Let $x \in X(B)$ be balanced. Using Lemma 4.4 we obtain a rational $y$ with denominator $K$, with the same zero coordinates as $x$ and such that $\left|x_{j}-y_{j}\right| \leqq$ $4 /(5 K), j=1, \cdots, 5$. We shall complete the proof by showing that $y$ is $3 / K$-balanced. If $y$ is not $3 / K$-balanced then there exist players $i, j \in B \in \mathbb{B}, i \neq j$, such that $y_{i}>0$ and $h_{i j}(y)=s_{i j}(y)-s_{j i}(y)<-3 / K$. Since $h_{i j}(y)$ is a rational number with denominator $K$, we must have $h_{i j}(y) \leqq-4 / K$. On the other hand, using Lemma 4.5, we have

$$
\begin{aligned}
h_{i j}(y) & =h_{i j}(x)+\left(h_{i j}(y)-h_{i j}(x)\right) \\
& \geqq h_{i j}(x)-\left|h_{i j}(y)-h_{i j}(x)\right| \geqq-\left|h_{i j}(y)-h_{i j}(x)\right| \\
& \geqq-4\|x-y\| \geqq-4 \cdot 4 /(5 K)>-4 / K
\end{aligned}
$$

a contradiction which shows that the assumption that $y$ is not $3 / K$-balanced is false.

5. Step A. Let $(N, v)$ be an integral five person game and $B$ a coalition structure. The problem that confronts us in step $\mathrm{A}$ is how to choose from all the rational points in $X(B)$, a set of points, with the same (not too large) denominator $K$, that will be a $\delta$-kernel with volume as small as possible. Given $K$, Lemma 4.4 and the subsequent remark tell us that the best possible $\delta$ is $\delta(K)=4 /(5 K)$. Having determined $\delta$ we have to decide upon the way of choosing the points, so as to obtain a $\delta(K)$-kernel with a minimum number of points. We do this by choosing all $\epsilon$-balanced points, where $\epsilon$ is determined by Lemma 4.7, i.e., $\epsilon=\epsilon(K)=3 / K$.

We can now describe step A formally. Let $K$ be a natural number and $\epsilon=3 / K$. Step A consists of finding all rational points in $X(B)$ with denominator $K$ that are $\epsilon$-balanced.

There remains only the problem of determining $K$. If $K$ is determined then so is $\epsilon$, and therefore the set of points that will pass step A is determined. In step B we shall have to check all the rational points with denominator not exceeding $K_{5}$, that are in a $\delta(K)$-neighborhood of this set. Increasing $K$ increases the number of points that must be checked in step A, but, on the other hand, decreases $\epsilon$, and therefore decreases the proportion that will pass step A; in addition $\delta(K)$ also decreases, so that we can expect that the "volume" we shall have to inspect in step $\mathrm{B}$ decreases. This conjecture was verified in the experiments that we performed. 
We carried out step A for various games and coalition structures, with denominators $K$ in the range 12-24, and observed that the number of points that passed step A was independent of $K$ in this range; we concluded that increasing $K$ decreases the time needed for step B. Clearly, increasing $K$ increases the time needed for step A itself. Now, what we want, is to minimize the total time needed for steps $\mathrm{A}$ and $\mathrm{B}$, so we have to find a compromise. A description of how this worked out for simple games may be found toward the end of $\$ 7$.

The number of points that passed step A varied greatly in the various experiments that we performed. We checked only characteristic functions $v$ and coalition structures $B$ where the values $v(B)$ for $B \in B$ were either 1 or 0 . For the denominator 24 we received from 4 to 240 points. The calculations in $\$ 4$ (see (4.3)) show that when $B=\{N\}$ and $v(N)=1$, the number of points that must be checked in step A is about 22000 , so that the proportion that passed step A did not exceed approximately one percent.

We shall now proceed to describe the computations. The data of step $A$ is the characteristic function $v$, the coalition structure $B$, the denominator $K$ and $\epsilon=$ $3 / K$. The computer multiplies $v$ and $\epsilon$ by $K$, so that in all the computations only integral numbers appear. The set $X(B)$ is thus transformed into $X_{\mathbf{K}}(B)=\{x$ : $x_{i} \geqq 0$ for $i=1, \cdots, 5$, and $\sum_{B} x_{i}=K v(B)$ for $\left.B \in B\right\}$. Then the computer examines all the integral points in $X_{K}(B)$, taken in lexicographic order; those points that satisfy equations (4.6) with $\epsilon=3$ are stored in the memory for use in step $B$.

The flow chart in Figure 1 describes the order of the computations for step A. All the expressions $e(S, x)$ are computed immediately after a point $x$ is generated. The functions $s_{i j}(x)$ are computed only when needed in the course of the check.

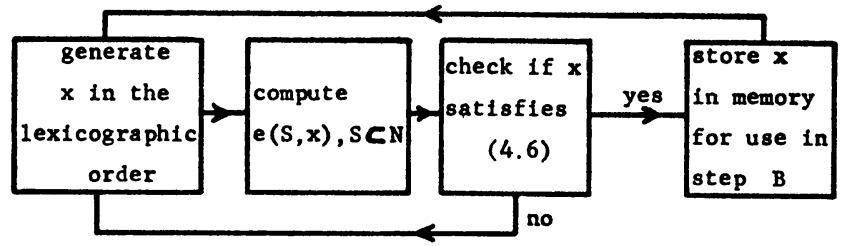

Figure 1. Order of computations for step A.

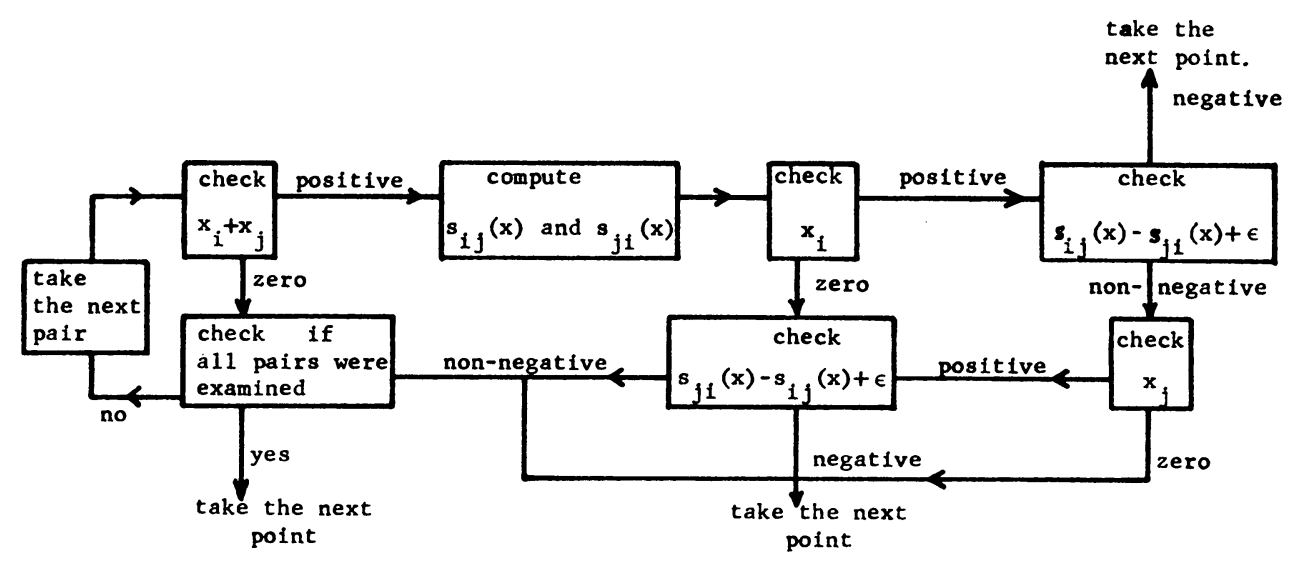

Figure 2. Check if $x$ satisfies (4.6). 
The check deserves special attention: it consists of comparing the functions $s_{i j}(x)$ and $s_{j i}(x)$, for all the pairs $\{i, j\}$ of different players that belong to the same coalition $B \in B$. The details are given in the flow chart in Figure 2.

When the point stored in the memory differs from its predecessor only in the last two coordinates, which is the case most frequently encountered, the expressions $e(S, x)$ are computed directly from those of the predecessor, in an easy way.

Another device to save time is the following: when a point is rejected, the check of the next point begins by examining the last pair that was inspected before, i.e. the pair that caused the rejection.

6. Step B. The data of step B consists of the data of Step A plus the list of the points that were found $3 / K$-balanced in step $\mathrm{A}$, where $K$ is the denominator of step A. These points form a set which is a $\delta(K)$-kernel. Step B consists of finding all the rational points with denominator not exceeding $K_{5}$ that are in the intersection of the kernel and the $\delta(K)$-neighborhood of the kernel determined by step A, and determining which of these points are extreme in the polyhedra defined by (3.2).

The new problems in step B are how to generate the points to be examined, and how to check extremality. The procedure of actually checking whether the point is in the kernel is the same as that of step A, except that now we examine if the points satisfy (3.1) and not (4.6).

The order of the computations is as follows. We take the denominators we have to examine in their natural order. When we reach a denominator $K_{1}$ we multiply $v$ by $K_{1}$, so that only integral numbers will appear in the computations. After the multiplication, the points that passed step $A$ are taken from the list, and for each point we examine all the integral points of $X_{K_{1}}(B)$ that are in a $\delta(K) K_{1}$-neighborhood of it, where $K$ is the denominator of step $\mathrm{A}$, and

$$
X_{K_{1}}(B)=\left\{x_{i}: x_{i} \geqq 0 \text { for } i=1, \cdots, 5 \text { and } \sum_{B} x_{i}=K_{1} v(B) \text { for } B \in \mathbb{B}\right\} .
$$

For a point taken from the list of step A, we compute first the bounds that define its $\delta(K) K_{1}$-neighborhood and afterwards we check all the integral points in the neighborhood, taken in the lexicographic order, for membership in the kernel.

Each point $x$ that emerges from this procedure is in the kernel. The next task is to check for extremality, i.e. to check whether $x$ is an extreme point of one of the polyhedra $C_{p}$ defined by (3.2). Now it is possible that $x$ is in more than one of these polyhedra. Let $E_{x}$ be the intersection of all the $C_{p}$ to which $x$ belongs. Certainly, if $x$ is extreme in one of the $C_{p}$, then it is extreme in $E_{x}$; the converse is also true. ${ }^{5}$ What we must therefore check is whether $x$ is extreme in $E_{x}$.

Among the linear inequalities $a \cdot y \geqq b$ defining $E_{x}$, some are satisfied as equalities by $x$; denote these by

$$
a_{1} \cdot y \geqq c_{1}, \quad a_{2} \cdot y \geqq c_{2}, \cdots, a_{m} \cdot y \geqq c_{m},
$$

where the $a_{i}$ are vectors depending on $x$ and the $c_{i}$ are scalars depending on $x$. Thus we have

$$
a_{1} \cdot x=c_{1}, \quad a_{2} \cdot x=c_{2}, \cdots, a_{m} \cdot x=c_{m} .
$$

${ }^{5}$ This follows from the fact that the intersection of any two $C_{p}$ is a face of both. 
The point $x$ is extreme in $E_{x}$ if and only if there are 5 linearly independent $a_{i}$, i.e. the rank of the matrix $A_{x}$ formed by the $a_{i}$ is 5 . To find the rank of $A_{x}$, we form a matrix $D(x)$ of zeros and ones as follows: to each pair of distinct players $i$ and $j$ in the same coalition $B \in \mathbb{B}$, there is a row $D_{(i j)}$ in the matrix. Let the coalitions ${ }^{6}$ in $T_{i j}$ be $S_{i j}^{1}, \cdots, S_{i j}^{8}$; then $D_{(i j)}=\left(d_{(i j)}^{1}, \cdots, d_{(i j)}^{8}\right)$, where $d_{(i j)}^{k}=0$ or 1 , according as $e\left(S_{i j}^{k}\right)<s_{i j}(x)$ or $e\left(S_{i j}^{k}\right)=s_{i j}(x)$. Consider the system of linear equations

$$
e\left(S_{i j}^{k}\right)=e\left(S_{j i}^{k^{\prime}}\right) \text {, }
$$

where $k, k^{\prime}, i, j$, range over values such that

$$
d_{(i j)}^{k}=d_{(j i)}^{k^{\prime}}=1 \text { and } s_{i j}(x)=s_{j i}(x) .
$$

This system is closely related to the system (6.1). More precisely, let $f_{i j}^{k}$ be the incidence vector ${ }^{7}$ of the coalition $S_{i j}^{k}$, and let $F_{x}$ be the matrix whose rows are the unit vectors $e^{i}$ for $i$ obeying $x_{i}=0$, the incidence vectors of the coalitions $B$ in the coalition structure $B$, and the vectors $f_{i j}^{k}-f_{j i}^{k^{\prime}}$, where $k, k^{\prime}, i, j$, range over values such that (6.2) holds; then $F_{x}$ has the same rank as $A_{x}$. The test of extremality that we use consists of finding the rank of $F_{x}$ for each $x$. Once a point $x$ has been found to be extreme (i.e. $F_{x}$ has been found to have rank 5), it is stored in the memory together with the matrix $D(x)$, until all the extreme points have been found.

There remains only the problem of grouping the extreme points according to the various polyhedra $C_{p}$ to which they belong. Now it can be proved that the kernel of a five person game is one-dimensional, i.e. consists of a finite union of points and line segments. So the only question we have to settle is which pairs of extreme points can be joined by a line segment in the kernel. Let $x$ and $y$ be extreme points; and let $D(x)$ and $D(y)$ be their matrices. Then there is a convex polyhedron $C_{p}$ that contains both $x$ and $y$, if and only if for each two players $i$ and $j$ belonging to the same coalition $B \in \mathbb{B}$, either $x_{i}=y_{i}=0$ or $\sum_{k=1}^{8} d_{(i j)}^{k}(x) d_{(i j)}^{k}(y)>0$. So the question of "pairing" can be solved by computing the scalar products of the corresponding rows of $D(x)$ and $D(y)$.

The output is a list of extreme points, together with a list of all pairs such that the line segment joining them is in the kernel; it gives a complete picture of the kernel.

7. Weighted Majority Games. We recall a number of definitions and elementary facts. A game $G=(N, v)$ is called superadditive if for every pair of disjoint coalitions $S$ and $T$, we have $v(S \cup T) \geqq v(S)+v(T)$; monotonic if for every pair of coalitions $S$ and $T$ such that $S \supset T$, we have $v(S) \geqq v(T)$; constant-sum if $v(S)+$ $v(N-S)=v(N)$ for all $S$. A simple game is one whose characteristic function takes the values 0 and 1 only; then $S$ is called winning if $v(S)=1$, and losing if $v(S)=0$. A weighted majority game is a simple game for which there exist $n$ nonnegative numbers $w_{1}, \cdots, w_{n}$, and a real number $q$ (usually non-negative) such that $S$ is winning if and only if $\sum_{s} w_{i} \geqq q$. Then $w_{1}, \cdots, w_{n}$ are called weights for the players, and $q$ is called a quota; $\left[q ; w_{1}, \cdots, w_{n}\right]$ is called a representation of the game, though often we will use it to refer to the game itself. The representation of

- Because $n=5$ there are exactly 8 coalitions in each $T_{i j}$.

7 The vector whose $r$ th member is 0 or 1 according as $r$ is or is not in $S_{i j}^{k}$. 
a given weighted majority game is in no sense unique. Two representations of the same game are called equivalent. A simple game is superadditive if and only if there is no pair of disjoint winning coalitions. A weighted majority game is always monotonic, but it need be neither superadditive nor constant-sum; for example, [3; 1,1 , $2,2]$ is neither. Of course, both superadditivity and constant-sumness are intrinsic properties, i.e. they depend on the game (characteristic function) and not on its representation.

A player $i$ in a game $(N, v)$ is called at least as desirable as another player $j$ (in symbols $i \succsim j)$ if $v(S \cup\{i\}) \geqq v(S \cup\{j\})$ for all $S$ containing neither $i$ nor $j$. If $i \succsim j$ and $j \succsim i$ then $i$ and $j$ are called symmetric $(i \sim j)$. Every weighted majority game has a representation $\left[q ; w_{1}, \cdots, w_{n}\right]$ that completely reflects the desirability relation, i.e. such that $i \succsim j$ if and only if $w_{i} \geqq w_{j}$; but not every representation need do this. Two games $(N, v)$ and $(M, u)$ are said to have the same desirability pattern if there is a one-one mapping $\varphi$ of $N$ onto $M$ that preserves desirability, i.e. so that $i \succsim j$ if and only if $\varphi(i) \succsim \varphi(j)$. The desirability pattern is an intrinsic property of the game.

A veto player $i$ in a simple game $(N, v)$ is a player such that any coalition $S$ not containing $i$ is losing. There may be more than one veto player in a game. A dummy is a player $i$ such that $v(S \cup\{i\})=v(S)$. If $i$ is a dummy in a weighted majority game then the game has a representation in which $w_{i}=0$.

In this section we tabulate the kernels of all weighted majority games with at most 5 players, for the coalition structure consisting of the set $N$ of all players only. From this table it is possible to compute the kernel of any superadditive weighted majority game (regardless of the number of players), for coalition structures in which the winning coalition ${ }^{8}$ has at most 5 players. We first describe the arrangement of the table, then show how to compute the kernels of any weighted majority game (with the above restriction) from it.

In the table, the games are identified by representations. Since the kernel is an intrinsic property of the game, each game appears in only one representation. However, a user of the table may wish to find the kernel of a game for which he has a representation that does not appear in the table. In order to simplify the task of finding an equivalent representation in the table, it is desirable, insofar as possible, to classify the games in the table by intrinsic properties. This is done in two ways. First, the table is divided into three parts: Games with veto players; superadditive games without veto players; and nonsuperadditive games without veto players (all three properties are intrinsic). Second, the games in the last two parts are classified by desirability pattern. Though the desirability pattern cannot always be read off at once from a given representation of a game, for a 5-person game it can usually be determined rather quickly.

We first describe the second and third parts, which are the more interesting. The first column of the table is a serial number (to simplify references to the table). The second column signifies the desirability pattern; the notation used is selfexplanatory. The symbol for the desirability pattern is printed only next to the first game with this pattern, to make the table easier to read. The third column gives a representation of the game. The kernel of the game, with coalition structure

\footnotetext{
${ }^{8}$ Because of superadditivity there is at most one.
} 
$N$, is given in the fourth column. The kernel usually consists of a point or an interval; there is one exception (game 5-71) in which it is V-shaped. When the kernel consists of a single point, the point is given; when it is an interval, the two endpoints are given; in the $\mathrm{V}$-shaped case, all three vertices are given, with the one common to the two intervals between the other two. A common denominator is signified by /; thus 12234/12 signifies the point (1/12, 1/6, 1/6, 1/4, 1/3).

In the first part, the games with veto players are listed and given serial numbers, but the kernels are not given nor are the games classified by desirability pattern. It can be shown that the kernel of a game with veto players (for coalition structure $\{N\}$ ) consists of a single point, in which the veto players share the amount 1 equally, and the remaining players get 0 , (Example: The kernel of $[5 ; 1,1,2,2]$ is $0011 / 2$.) Thus the kernel can be determined immediately as soon as the game is given, and there is no need for tabulation. The list is included for the secondary purpose of completing the list of weighted majority games with at most 5 players.

Games with dummies are excluded. A weighted majority game in which player 1 is a dummy has a representation of the form $\left[q ; 0, w_{2}, \cdots, w_{n}\right]$, and its kernel is the set of all payoff vectors of the form $(0, x)$, where $x$ is in the kernel of $\left[q ; w_{2}\right.$, $\left.\cdots, w_{n}\right]$.

To show how the table can be used in computing the kernels of superadditive weighted majority games for coalition structures other than $\{N\}$, we now discuss the notions of "pseudo-kernel" and "reduced game" due to Davis and Maschler $[3, \S 7]$. Suppose $(N, v)$ is a game that does not necessarily satisfy the normalization assumptions $v(\{i\})=0$; as we will soon see, such games arise naturally in the process of computing the kernel of normalized games for certain coalition structures other than $\{N\}$. The kernel of a non-normalized game $(N, v)$ is defined by renormalizing. More precisely, define a game $(N, u)$ by $u(S)=v(S)-\sum_{B} v(\{i\})$, and let $K$ be the kernel of $(N, u)$ for coalition structure $B$; then the kernel of $(N, v)$ for $B$ is defined to be $K+(v(\{1\}), \cdots, v(\{n\}))$. Equivalently, the kernel of $(N, v)$ may be defined by replacing the condition $x_{j}>0$ in the definition by $x_{j}>$ $v(\{j\})$. As it happens, though, this is not the definition that is appropriate for our current purpose. What is needed here is the pseudo-kernel, which is defined in literally the same way as in Section 2, retaining the condition $x_{j}>0$ even though $v(\{j\})$ may differ from 0 .

To explain the notion of reduced game, we need the following notational device: If $x$ is a payoff vector to the player set $N$, and if $B \subset N$, denote the vector $\left\{x_{i}\right\}_{i \& B}$ of payoffs to $B$ by $x_{B}$. If $x$ is viewed as a function from $N$ to the reals, then $x_{B}$ is the restriction of $x$ to $B$.

Now let $(N, v)$ be a monotonic (normalized) game, $B=\left\{B_{1}, \cdots, B_{k}\right\}$ a coalition structure such that $v\left(B_{2}\right)=\cdots=v\left(B_{k}\right)=0$. Define the reduced game $\left(B_{1}, v^{*}\right)$ by $v^{*}\left(B_{1}\right)=v(B)$ and $v^{*}(B)=v\left(B \cup B_{2} \cup \ldots \cup B_{k}\right)$ for all $B$ that are strictly included in $B_{1}$; the game $\left(B_{1}, v^{*}\right)$ will in general be neither monotonic nor normalized. It is now easily seen that $x$ is in the kernel of $(N, v)$ for coalition structure $B$ if and only if $x_{i}=0$ for $i \notin B_{1}$ and $x_{B_{1}}$ is in the pseudo-kernel of $\left(B_{1}, v^{*}\right)$ for coalition structure $\left\{B_{1}\right\}$. Of course, if $\left(B_{1}, v^{*}\right)$ happens to be normalized, then its pseudo-kernel and its true kernel coincide.

Suppose now that $\left[q ; w_{1}, \cdots, w_{n}\right]$ is a superadditive weighted majority game, $B$ a coalition structure. Because of superadditivity there can be at most one winning coalition in $B$. If there are none the kernel is trivial; assume therefore that $B$ is 
the unique winning coalition. Then the reduced game corresponding to the structure $B$ and the coalition $B$ is the game $\left[q-\sum_{i \neq B} w_{i} ; w_{B}\right]$. The reduced game may or may not have some players whose quotas $w_{i}$ exceed the reduced quota $q-$ $\sum_{i \notin B} w_{i}$. If it does, then those players will win by themselves, so that the reduced game will not be normalized. In either case, the kernel of the original game for the coalition structure $B$ can be deduced from the pseudo-kernel of the reduced game for coalition structure $\{B\}$, with the understanding that the pseudo-kernel coincides with the kernel when the reduced game is normalized.

For example, if we wish to find the kernel of $[4 ; 1,1,2,2]$ for the coalition structure $(123,4)$, we are led to the reduced game $[2 ; 1,1,2]$, in which player 3 wins by himself. The pseudo-kernel of the latter game for the coalition structure $\{123\}$ is $112 / 4$, so that the kernel of the original game for $\{123,4\}$ is $1120 / 4$. The true kernel of $[2 ; 1,1,2]$ for $\{123\}$ is 001 , but this is irrelevant.

The pseudo-kernels of weighted majority games with at most 5 players and at least 1 winning player are listed in the fourth part of the table. By using the entire table and the technique described above, one can obtain the kernel of any superadditive weighted majority game, with arbitrarily many players, for coalition structures in which the winning coalition has at most 5 players.

The remarks made above about kernels of games with dummies apply also to pseudo-kernels.

Example 7.3. Calculate the kernel of $[7 ; 1,2,2,3,3]$ (game no. 5-41) for all coalition structures.

Solution. It is necessary to consider only coalition structures of the form $\{B, N-B\}$, where $B$ is winning. The winning coalitions are $12345,2345,1345$, $1234,145,234,345$, and coalitions obtained from these by replacing players by symmetric players. For $B=N$ the kernel can be found by looking at the table. For $B=\{2345\}$ we obtain $\left[7-w_{1} ; 2,2,3,3\right]=[6 ; 2,2,3,3]$. This representation does not appear in the table. To find an equivalent representation in the table, we note that it is superadditive and that its desirability pattern is $a a b b$. The only game with these specifications is $[4 ; 1,1,2,2]$ (no. 4-8), and its kernel is $1122 / 6$. Hence the kernel of our game for coalition structure $\{1,2345\}$ is $01122 / 6$. For the coalition structure $\{1234,5\}$, the reduced game is $[4 ; 1,2,2,3]$. This representation appears in the table itself (no. 4-12), and so we obtain 12230/8 for the kernel. For $\{145,23\}$, the reduced game is $[3 ; 1,3,3]$. This is a non-normalized game in which the first player is a dummy. Removing the dummy, we obtain $[3 ; 3,3]$, which is equivalent to $[1 ; 1,1]$ (game no. 2-2). Hence the pseudo-kernel of $[3 ; 1,3,3]$ is $011 / 2$, and that of the original game for the coalition structure $\{145,23\}$ is $00011 / 2$. The kernels for the other coalition structures are similarly obtained. The final result is as follows:

Coalition structure

12345

2345,1

1345,2

1234,5

145,23

234,15

345,12
Kernel

$01122 / 6$

$* 1122 / 6$

$0 * 111 / 3$

$1223 * / 8$

$0 * * 11 / 2$

** $112 * / 4$

$* * 111 / 3$ 
TABLE 1. Weighted Majority Games With at Most 5 Players and Their Kernels

\begin{tabular}{|c|c|c|c|c|c|}
\hline $\begin{array}{l}\text { Serial } \\
\text { num- } \\
\text { ber }\end{array}$ & Game & $\begin{array}{c}\text { Serial } \\
\text { num- } \\
\text { ber }\end{array}$ & Game & $\begin{array}{c}\text { Serial } \\
\text { num- } \\
\text { ber }\end{array}$ & Game \\
\hline
\end{tabular}

1. Games with Veto Players

\begin{tabular}{|c|c|c|c|c|c|c|}
\hline $\begin{array}{l}2-1 \\
3-1 \\
3-2 \\
4-1 \\
4-2 \\
4-3 \\
4-4 \\
4-5\end{array}$ & $\begin{array}{l}{[2 ;} \\
{[3 ;} \\
{[3 ;} \\
{[4 ;} \\
{[4 ;} \\
{[4 ;} \\
{[5 ;} \\
{[5 ;}\end{array}$ & $\begin{array}{l}1] \\
1,1] \\
1,2] \\
1,1,1] \\
1,1,2] \\
1,1,3] \\
1,2,2] \\
1,2,3]\end{array}$ & $\begin{array}{l}5-1 \\
5-2 \\
5-3 \\
5-4 \\
5-5 \\
5-6 \\
5-7 \\
5-8 \\
5-9\end{array}$ & $\begin{array}{l}{[5 ; 1,1,1,1,1]} \\
{[5 ; 1,1,1,1,2]} \\
{[5 ; 1,1,1,1,3]} \\
{[5 ; 1,1,1,1,4]} \\
{[6 ; 1,1,1,2,2]} \\
{[7 ; 1,1,1,3,3]} \\
{[6 ; 1,1,1,2,3]} \\
{[6 ; 1,1,1,2,4]} \\
{[7 ; 1,1,1,3,4]}\end{array}$ & $\begin{array}{l}5-10 \\
5-11 \\
5-12 \\
5-13 \\
5-14 \\
5-15 \\
5-16 \\
5-17\end{array}$ & $\begin{array}{l}{[7 ; 1,1,2,2,2]} \\
{[7 ; 1,1,2,2,3]} \\
{[7 ; 1,1,2,2,4]} \\
{[7 ; 1,1,2,2,5]} \\
{[8 ; 1,1,2,3,3]} \\
{[8 ; 1,1,2,3,5]} \\
{[9 ; 1,2,2,3,4]} \\
{[9 ; 1,2,2,3,5]}\end{array}$ \\
\hline \multicolumn{2}{|c|}{$\begin{array}{c}\text { Serial } \\
\text { number }\end{array}$} & \multicolumn{2}{|c|}{$\begin{array}{l}\text { Desirability } \\
\text { pattern }\end{array}$} & \multicolumn{2}{|l|}{ Game } & Kernel \\
\hline
\end{tabular}

2. Super additive Games without Veto Players

\begin{tabular}{|c|c|c|c|}
\hline $\begin{array}{l}3-3 \\
4-6 \\
4-7 \\
4-8 \\
4-9 \\
5-18 \\
5-19 \\
5-20 \\
5-21 \\
5-22 \\
5-23 \\
5-24 \\
5-25 \\
5-26 \\
5-27 \\
5-28 \\
5-29 \\
5-30 \\
5-31 \\
5-32 \\
5-33 \\
5-34 \\
5-35 \\
5-36 \\
5-37 \\
5-38 \\
5-39 \\
5-40\end{array}$ & $\begin{array}{l}\text { aaa } \\
\text { aaaa } \\
\text { aaab } \\
\text { aabb } \\
\text { abbc } \\
\text { aaaaa } \\
\text { aaaab } \\
\text { aaabb }\end{array}$ & $\begin{array}{l}{[2 ; 1,1,1]} \\
{[3 ; 1,1,1,1]} \\
{[3 ; 1,1,1,2]} \\
{[4 ; 1,1,2,2]} \\
{[5 ; 1,2,2,3]} \\
{[3 ; 1,1,1,1,1]} \\
{[4 ; 1,1,1,1,1]} \\
{[4 ; 1,1,1,1,2]} \\
{[4 ; 1,1,1,1,3]} \\
{[4 ; 1,1,1,2,2]} \\
\\
{[5 ; 1,1,1,2,2]} \\
{[6 ; 1,1,1,3,3]} \\
{[7 ; 2,2,2,3,3]} \\
\\
{[5 ; 1,1,1,2,3]} \\
{[5 ; 1,1,2,2,2]} \\
\\
{[6 ; 1,1,2,2,2]} \\
{[5 ; 1,1,2,2,3]} \\
{[6 ; 1,1,2,2,3]} \\
\\
{[6 ; 1,1,2,2,4]} \\
{[7 ; 1,1,3,3,4]} \\
{[8 ; 1,1,3,3,5]} \\
{[8 ; 2,2,3,3,4]} \\
{[6 ; 1,1,2,3,3]} \\
{[7 ; 1,1,2,3,4]} \\
{[9 ; 2,2,3,4,5]} \\
{[6 ; 1,2,2,2,3]} \\
{[7 ; 1,2,2,2,3]} \\
{[7 ; 1,2,2,2,5]}\end{array}$ & $\begin{array}{l}111 / 3 \\
1111 / 4 \\
1112 / 5 \\
1122 / 6 \\
0111 / 3 \\
11111 / 5 \\
11111 / 5 \\
11112 / 6 \\
11113 / 7 \\
22244 / 14 \\
00077 / 14 \\
11122 / 7 \\
11133 / 9 \\
22222 / 10 \\
00055 / 10 \\
11123 / 8 \\
33333 / 15 \\
00555 / 15 \\
11222 / 8 \\
11223 / 9 \\
11112 / 6 \\
00222 / 6 \\
11224 / 10 \\
00111 / 3 \\
00111 / 3 \\
11111 / 5 \\
00111 / 3 \\
00111 / 3 \\
11122 / 7 \\
12223 / 10 \\
01111 / 4 \\
01112 / 5\end{array}$ \\
\hline
\end{tabular}


TABLE 1-Continued

\begin{tabular}{c|c|c|c}
\hline $\begin{array}{c}\text { Serial } \\
\text { number }\end{array}$ & $\begin{array}{c}\text { Desirability } \\
\text { pattern }\end{array}$ & Game & Kernel \\
\hline \multicolumn{2}{c}{ 2. Superadditive Games without Veto Players-Continued } \\
\hline $5-41$ & abbcc & {$[7 ; 1,2,2,3,3]$} & $01122 / 6$ \\
$5-42$ & abbcd & {$[8 ; 1,2,2,3,3]$} & $01111 / 4$ \\
$5-43$ & & {$[7 ; 1,2,2,3,4]$} & $01112 / 5$ \\
$5-44$ & abccd & {$[8 ; 1,2,2,3,4]$} & $12234 / 12$ \\
$5-45$ & abcde & {$[8 ; 1,2,2,4,5]$} & $01122 / 6$ \\
$5-46$ & {$[8 ; 1,2,3,3,5]$} & $00111 / 3$ \\
$5-47$ & {$[9 ; 1,2,3,4,5]$} & $01112 / 5$ \\
$5-48$ & abs & $00111 / 3$ \\
\hline
\end{tabular}

3. Nonsuperadditive Games

\begin{tabular}{|c|c|c|c|}
\hline $\begin{array}{l}4-10 \\
4-11\end{array}$ & $\begin{array}{l}\text { aaaa } \\
\text { aabb }\end{array}$ & $\begin{array}{l}{[2 ; 1,1,1,1]} \\
{[3 ; 1,1,2,2]}\end{array}$ & $\begin{array}{l}1111 / 4 \\
1111 / 4\end{array}$ \\
\hline $\begin{array}{l}4-12 \\
5-49 \\
5-50 \\
5-51\end{array}$ & $\begin{array}{l}\text { abbc } \\
\text { aaaaa } \\
\text { aaaab } \\
\text { aaabb }\end{array}$ & $\begin{array}{l}{[4 ; 1,2,2,3]} \\
{[2 ; 1,1,1,1,1]} \\
{[3 ; 1,1,1,1,2]} \\
{[3 ; 1,1,1,2,2]}\end{array}$ & $\begin{array}{l}1223 / 8 \\
11111 / 5 \\
11112 / 6 \\
11122 / 7\end{array}$ \\
\hline $5-52$ & & {$[4 ; 1,1,1,3,3]$} & $\begin{array}{l}11111 / 5 \\
22222 / 10\end{array}$ \\
\hline $\begin{array}{l}5-53 \\
5-54\end{array}$ & aaabc & $\begin{array}{l}{[6 ; 2,2,2,3,3]} \\
{[4 ; 1,1,1,2,3]}\end{array}$ & $\begin{array}{l}22233 / 12 \\
11112 / 6\end{array}$ \\
\hline $5-55$ & aabbb & {$[3 ; 1,1,2,2,2]$} & $\begin{array}{l}33333 / 15 \\
00555 / 15\end{array}$ \\
\hline $\begin{array}{l}5-56 \\
5-57 \\
5-58\end{array}$ & aabbc & $\begin{array}{l}{[4 ; 1,1,2,2,2]} \\
{[4 ; 1,1,2,2,3]} \\
{[5 ; 1,1,2,2,4]}\end{array}$ & $\begin{array}{l}11222 / 8 \\
11223 / 9 \\
00336 / 12 \\
22224 / 12\end{array}$ \\
\hline $\begin{array}{l}5-59 \\
5-60 \\
5-61\end{array}$ & & $\begin{array}{l}{[6 ; 1,1,3,3,4]} \\
{[6 ; 1,1,3,3,5]} \\
{[7 ; 2,2,3,3,4]}\end{array}$ & $\begin{array}{l}11334 / 12 \\
00112 / 4 \\
44448 / 24 \\
33666 / 24\end{array}$ \\
\hline $5-62$ & aabcc & {$[5 ; 1,1,2,3,3]$} & $\begin{array}{l}00044 / 8 \\
11222 / 8\end{array}$ \\
\hline $\begin{array}{l}5-63 \\
5-64\end{array}$ & aabcd & $\begin{array}{l}{[5 ; 1,1,2,3,4]} \\
{[8 ; 2,2,3,4,5]}\end{array}$ & $\begin{array}{l}00011 / 2 \\
00088 / 16 \\
22345 / 16\end{array}$ \\
\hline $\begin{array}{l}5-65 \\
5-66\end{array}$ & abbbc & $\begin{array}{l}{[4 ; 1,2,2,2,3]} \\
{[5 ; 1,2,2,2,3]}\end{array}$ & $\begin{array}{l}12223 / 10 \\
03333 / 12 \\
22224 / 12\end{array}$ \\
\hline $\begin{array}{l}5-67 \\
5-68 \\
5-69 \\
5-70 \\
5-71\end{array}$ & $\begin{array}{l}\text { abbcc } \\
\text { abbcd }\end{array}$ & $\begin{array}{l}{[6 ; 1,2,2,2,5]} \\
{[4 ; 1,2,2,3,3]} \\
{[5 ; 1,2,2,3,3]} \\
{[5 ; 1,2,2,3,4]} \\
{[6 ; 1,2,2,3,4]}\end{array}$ & $\begin{array}{l}12225 / 12 \\
11111 / 5 \\
01111 / 4 \\
11122 / 7 \\
03333 / 12 \\
12234 / 12 \\
00066 / 12\end{array}$ \\
\hline
\end{tabular}


TABLE 1-Continued

\begin{tabular}{|c|c|c|c|}
\hline $\begin{array}{c}\text { Serial } \\
\text { number }\end{array}$ & $\begin{array}{l}\text { Desirability } \\
\text { pattern }\end{array}$ & Game & Kernel \\
\hline \multicolumn{4}{|c|}{ 3. Nonsuperadditive Games-Continued } \\
\hline $\begin{array}{l}5-72 \\
5-73 \\
5-74 \\
5-75\end{array}$ & $\begin{array}{l}\text { abccd } \\
\text { abcde }\end{array}$ & $\begin{array}{l}{[6 ; 1,2,2,4,5]} \\
{[6 ; 1,2,3,3,4]} \\
{[7 ; 1,2,3,3,5]} \\
{[7 ; 1,2,3,4,5]}\end{array}$ & $\begin{array}{l}00011 / 2 \\
01111 / 4 \\
12335 / 14 \\
00022 / 4 \\
01111 / 4\end{array}$ \\
\hline $\begin{array}{c}\text { Serial } \\
\text { number }\end{array}$ & $\begin{array}{l}\text { Desirability } \\
\text { pattern }\end{array}$ & Game & Pseudo-kernel \\
\hline
\end{tabular}

4. Games with Winning Players and Their Pseudo-Kernels

\begin{tabular}{|c|c|c|c|}
\hline $\begin{array}{l}1-1 \\
2-2 \\
3-4 \\
3-5 \\
4-13 \\
4-14 \\
4-15 \\
4-16 \\
4-17 \\
5-76 \\
5-77 \\
5-78 \\
5-79 \\
5-80 \\
5-81 \\
5-82 \\
5-83 \\
5-84 \\
5-85 \\
5-86 \\
\\
5-87 \\
5-88 \\
5-89 \\
5-90 \\
5-91 \\
5-92\end{array}$ & $\begin{array}{l}\text { a } \\
\text { aa } \\
\text { aaa } \\
\text { aab } \\
\text { aaaa } \\
\text { aaab } \\
\text { aabb } \\
\text { aabc } \\
\text { aaaaa } \\
\text { aaaab } \\
\text { aaabb } \\
\text { aaabc } \\
\text { aabbb } \\
\text { aabbc }\end{array}$ & $\begin{array}{l}{[1 ; 1]} \\
{[1 ; 1,1]} \\
{[1 ; 1,1,1]} \\
{[2 ; 1,1,2]} \\
{[1 ; 1,1,1,1]} \\
{[2 ; 1,1,1,2]} \\
{[3 ; 1,1,1,3]} \\
{[2 ; 1,1,2,2]} \\
{[3 ; 1,1,2,3]} \\
{[1 ; 1,1,1,1,1]} \\
{[2 ; 1,1,1,1,2]} \\
{[3 ; 1,1,1,1,3]} \\
{[4 ; 1,1,1,1,4]} \\
{[2 ; 1,1,1,2,2]} \\
{[3 ; 1,1,1,3,3]} \\
{[3 ; 1,1,1,2,3]} \\
{[4 ; 1,1,1,2,4]} \\
{[4 ; 1,1,1,3,4]} \\
{[2 ; 1,1,2,2,2]} \\
{[3 ; 1,1,2,2,3]} \\
{[4} \\
{[4 ; 1,1,2,2,4]} \\
{[5 ; 1,1,2,2,5]} \\
{[3 ; 1,1,2,3,3]} \\
{[5 ; 1,1,2,3,5]} \\
{[4 ; 1,2,2,3,4]} \\
{[5 ; 1,2,2,3,5]}\end{array}$ & $\begin{array}{l}1 / 1 \\
11 / 2 \\
111 / 3 \\
112 / 4 \\
1111 / 4 \\
1112 / 5 \\
1113 / 6 \\
1122 / 6 \\
0011 / 2 \\
11111 / 5 \\
11112 / 6 \\
11113 / 7 \\
11114 / 8 \\
11122 / 7 \\
11133 / 9 \\
11123 / 8 \\
00011 / 2 \\
00011 / 2 \\
11222 / 8 \\
00222 / 6 \\
11112 / 6 \\
11224 / 10 \\
00112 / 4 \\
00111 / 3 \\
00011 / 2 \\
12234 / 12 \\
01112 / 5\end{array}$ \\
\hline
\end{tabular}

The stars indicate players not in the winning coalition of the coalition structure, who therefore automatically get 0 (as distinguished from players who are in the winning coalition, but get 0 anyway).

The authors have tabulated the kernels of all superadditive 5-person games for all coalition structures, by the above procedure. The table is available from them upon request.

We remark that all kernels and pseudo-kernels appearing in the table appear as 
the kernel of some 6-person superadditive game, with an appropriate coalition structure.

The computations of the kernels in Table 1 were carried out on the CDC-1604-A. Trial and error led to the conclusion that the denominator 24 is fairly efficient for step A. With this denominator, the time needed for the entire calculation of the kernel of a simple game (both steps) is approximately 55 seconds (the addition time of the CDC-1604-A is 7.2 $\mu$ sec.). Of this, step A takes 18-27 seconds, and step $\mathrm{B}$ the remainder.

To minimize the human factor in the computation of the table, and consequent mistakes, a computer program was written to compute the characteristic function of a weighted majority game from its representation. It was therefore possible to use the representations as input to the computer.

A word is in order about the compilation of the games that appear in the table. For games with up to four players we used Shapley's list [14]. For games with 5 players, we used Isbell's list [7] of 6- and 7-person superadditive constant-sum weighted majority games. The work was divided into two steps:

(i) Deriving all the 6-person superadditive weighted majority games from Isbell's list.

(ii) Deriving all the 5-person weighted majority games from the 6-person superadditive ones.

To accomplish step (i), we made use of the "zero-sum $(n+1)$-person extension" of an $n$-person game due to von Neumann and Morgenstern ${ }^{9}[10$, p. 506]. The zero-sum extension is always unique. For weighted majority games, if $\left[q ; w_{1}, \cdots, w_{n+1}\right]$ is zero-sum, then it is the zero-sum extension of $\left[q ; w_{1}, \cdots\right.$, $w_{j-1}, w_{j+1}, \cdots, w_{n+1}$ ( which is always superadditive) for every $j$; and conversely, if $\left[q ; w_{1}, \cdots, w_{n}\right]$ is superadditive, then there is a $w$ (not necessarily $\geqq w_{n}$ ) such that $\left[q ; w_{1}, \cdots, w_{n}, w\right]$ is its zero-sum extension. Step (i) consisted of compiling all the 6-person games $\left[q ; w_{1}, \cdots, w_{j-1}, w_{j+1}, \cdots, w_{7}\right]$ for each $\left[q ; w_{1}, \cdots, w_{7}\right]$ in Isbell's list and each $j$ between 1 and 7, and adding to them the games in Isbell's list of 6-person games. It can be deduced from the fact that the "zero-sum extension" is defined in an intrinsic way that this yields all the 6-person weighted majority superadditive games.

To accomplish step (ii), we made use of the Maschler-Davis "reduced game" (defined above); this too is an intrinsic process. Our procedure was to compile all the 5-person games $\left[q-w_{j} ; w_{1}, \cdots, w_{j-1}, w_{j+1}, \cdots, w_{6}\right]$ for all $\left[q ; w_{1}, \cdots, w_{6}\right]$ resulting from step (i) and all $j$ from 1 to 6 . This yields all weighted majority 5person games.

Steps (i) and (ii) were programmed on the CDC-1604-A. Of course the final list contained many duplications. Duplications due to multiple appearances of the same representation were removed first; then the characteristic function of each representation was computed and duplications due to a characteristic function having different representations were removed.

8. Extreme Games. For a fixed player set $N$ with $n$ members, the set of all superadditive games $G=(N, v)$ may be considered a cone in euclidean space of

${ }^{9}$ The "zero-sum extension" of the superadditive game $(N, v)$ is the game $(N \cup\{n+1\}, w)$, where $w(S)=v(S)$ for $n+1 \notin S$ and $w(S)=v(N)-v(N-S)$ for $n+1 \in S$. 
dimension $2^{n}-n-1\left(2^{n}\right.$ is the number of coalitions, and $v$ is fixed on the empty coalition and on the 1-member coalitions). A game $(N, v)$ is called extreme if it is on an extreme ray of this cone; that is, if $v$ is not the sum of two unproportional superadditive characteristic functions. All superadditive simple games are extreme.

Constant-sum extreme games have received particular attention, in papers by Griesmer [5] and Gurk [6]. When $n \leqq 4$ all constant-sum extreme games are simple; however, when $n \geqq 5$ there are non-simple constant-sum extreme games as well. Gurk [6] found a method of determining all constant-sum extreme games with five players; it turns out that in addition to the simple ones, there are 8 essentially different such games. ${ }^{10}$ In all these games the characteristic function $v$ takes exactly three values-in the normalization we adopt here, they are $0,1 / 2$, and 1 .

We have constructed these 8 games by Gurk's method, and computed their kernels, for all coalition structures, with the program described in the previous sections; the results are tabulated in Table 2. For general integral games, the computer time needed for the computation of the kernel corresponding to a coalition structure $B$ depends monotonically on the values of $v$, and in particular on the $v(B)$ for $B$ in $B$. In the integral games corresponding to the 8 extreme games under consideration, $v$ takes the values 0,1 , and 2 ; after simple games, therefore, these may be expected to be the games whose kernels may be most quickly computed. As it turned out, the time needed for the coalition structure containing the allplayer coalition only was usually about 4 or 5 minutes (compared with about 1 minute for simple games). For other coalition structures, the computation time was a matter of seconds.

From the theoretical viewpoint, the kernels tabulated here are interesting because there are coalition structures in which more than one coalition gets positive payoff; for superadditive simple games, this is impossible.

We now describe the construction of the table and its use. Each of the eight games is identified by the set of 3-person coalitions whose value is 1 ; the value of each of the remaining 3-person coalitions is $1 / 2$. These conditions determine a unique constant-sum 5-person game such that $v(N)=1$.

For each game there are two columns; in the left column there is a list of coalition structures, and in the right column the kernels corresponding to these coalition structures are tabulated. In no case do we list all the coalition structures; in the next paragraph we will discuss how the kernels corresponding to the unlisted coalition structures may be obtained. When the kernel consists of a single point, the point is given; when it is an interval, the two end-points are given; when it is Vshaped, all three vertices are given, with the one common to the two intervals between the other two. In this table we do not use the "common denominator" notation adopted in $\$ 7$. When a coalition consists of a single player, that player necessarily gets 0 in the kernel; in that case his payoff in the kernel is indicated by a star rather than a 0 . When there is more than one non- flat $^{11}$ coalition in a structure, then one of these is picked arbitrarily and its members (in the left column) and payoffs (in the right column) are italicized. Thus the coalition structure in

${ }^{10}$ I.e. every 5-person non-simple constant-sum extreme game is obtained from one of these eight by permuting the players and multiplying the characteristic function by a non-negative constant.

${ }^{11}$ A coalition $S$ is fat if $v(S)=0$. 
TABLE 2

Kernels of Five Person Constant-Sum Non-Simple Extreme Games

\begin{tabular}{|c|c|c|c|c|c|c|c|c|c|c|c|}
\hline $\begin{array}{l}\text { Coalition } \\
\text { structure }\end{array}$ & \multicolumn{5}{|c|}{ Kernel } & $\begin{array}{l}\text { Coalition } \\
\text { structure }\end{array}$ & \multicolumn{5}{|c|}{ Kernel } \\
\hline \multicolumn{6}{|c|}{$\begin{array}{c}\text { Game No. } 1 \\
v(S)=1 / 2 \text { for all } S \text { with } 3 \text { members }\end{array}$} & \multicolumn{6}{|c|}{$\begin{array}{c}\text { Game No. } 6 \\
v(\{1,2,3\})=v(\{3,4,5\})=v(\{1,2,5\})=1 \\
\end{array}$} \\
\hline $\begin{array}{l}12345 \\
1234,5 \\
123,45 \\
12,34,5\end{array}$ & $\begin{array}{l}1 / 5 \\
1 / 4 \\
1 / 6 \\
1 / 4\end{array}$ & \begin{tabular}{|l|}
$1 / 5$ \\
$1 / 4$ \\
$1 / 6$ \\
$1 / 4$
\end{tabular} & $\begin{array}{l}1 / 5 \\
1 / 4 \\
1 / 6 \\
1 / 4\end{array}$ & $\begin{array}{l}1 / 5 \\
1 / 4 \\
1 / 4 \\
1 / 4\end{array}$ & $\begin{array}{c}1 / 5 \\
* \\
1 / 4 \\
*\end{array}$ & $\begin{array}{l}12345 \\
1,2345 \\
3,1245 \\
4,1235 \\
124\end{array}$ & $\begin{array}{l}1 / 6 \\
* \\
1 / 4 \\
1 / 6 \\
3 / 16\end{array}$ & $\begin{array}{l}1 / 6 \\
3 / 8 \\
1 / 4 \\
1 / 6 \\
3 / 16\end{array}$ & $\begin{array}{l}1 / 3 \\
1 / 4 \\
* \\
1 / 3\end{array}$ & $\begin{array}{r}0 \\
1 / 8 \\
1 / 4 \\
* \\
1 / 8\end{array}$ & $\begin{array}{l}1 / 3 \\
1 / 4 \\
1 / 4 \\
1 / 3 \\
1 / 4\end{array}$ \\
\hline \multicolumn{6}{|c|}{$\begin{array}{c}\text { Game No. } 2 \\
v(\{2,3,4\})=1\end{array}$} & $\begin{array}{l}134,25 \\
135,24\end{array}$ & $\begin{array}{l}1 / 6 \\
1 / 2 \\
0\end{array}$ & $\begin{array}{l}1 / 6 \\
0 \\
3 / 8\end{array}$ & $\begin{array}{c}1 / 4 \\
1 / 3 \\
0 \\
1 / 4\end{array}$ & $\begin{array}{r}0 \\
1 / 2 \\
1 / 8\end{array}$ & $\begin{array}{c}1 / 4 \\
1 / 3 \\
0 \\
1 / 4\end{array}$ \\
\hline \multirow{2}{*}{$\begin{array}{l}12345 \\
1,2345 \\
2,1345 \\
123,45 \\
125,34 \\
1,23,45 \\
3,12,45\end{array}$} & \multirow{2}{*}{$\begin{array}{c}1 / 8 \\
* \\
1 / 6 \\
0 \\
1 / 8 \\
* \\
1 / 6\end{array}$} & \multirow{2}{*}{$\begin{array}{c}1 / 4 \\
1 / 4 \\
* \\
1 / 4 \\
1 / 4 \\
1 / 4 \\
1 / 3\end{array}$} & \multirow{2}{*}{$\begin{array}{l}1 / 4 \\
1 / 4 \\
1 / 3 \\
1 / 4 \\
1 / 4 \\
1 / 4 \\
*\end{array}$} & \multirow{2}{*}{$\begin{array}{l}1 / 4 \\
1 / 4 \\
1 / 3 \\
1 / 4 \\
1 / 4 \\
1 / 4 \\
1 / 3\end{array}$} & \multirow{2}{*}{$\begin{array}{l}1 / 8 \\
1 / 4 \\
1 / 6 \\
1 / 4 \\
1 / 8 \\
1 / 4 \\
1 / 6\end{array}$} & $\begin{array}{l}1,24,35 \\
3,15,24 \\
4,13,25\end{array}$ & $\begin{array}{c}* \\
0 \\
1 / 2 \\
1 / 6\end{array}$ & $\begin{array}{c}3 / 8 \\
1 / 2 \\
0 \\
1 / 6\end{array}$ & $\begin{array}{r}1 / 4 \\
* \\
* \\
1 / 3\end{array}$ & $\begin{array}{c}1 / 8 \\
0 \\
1 / 2 \\
*\end{array}$ & $\begin{array}{c}1 / 4 \\
1 / 2 \\
0 \\
1 / 8\end{array}$ \\
\hline & & & & & & \multicolumn{6}{|c|}{$\begin{array}{c}\text { Game No. } 7 \\
v(\{1,2,3)\}=v(\{2,3,4\})=v(\{3,4,5\})= \\
v(\{1,4,5\})=1\end{array}$} \\
\hline \multicolumn{6}{|c|}{$\begin{array}{c}\text { Game No. } \$ \\
v(\{2,3,4\})=v(\{3,4,5\})=1\end{array}$} & \multirow{3}{*}{$\begin{array}{l}12345 \\
1,2345 \\
2,1345 \\
3,1245 \\
134,25 \\
\\
135,24 \\
235,14 \\
125,34 \\
1,24,35 \\
2,14,95 \\
3,14,25 \\
1,25,94\end{array}$} & \multirow{3}{*}{$\begin{array}{c}2 / 7 \\
* \\
1 / 4 \\
1 / 6 \\
0 \\
1 / 6 \\
0 \\
0 \\
1 / 4 \\
1 / 4 \\
1 / 4 \\
* \\
* \\
1 / 4 \\
0 \\
1 / 4 \\
* \\
*\end{array}$} & \multirow{3}{*}{$\begin{array}{c}1 / 14 \\
1 / 4 \\
* \\
1 / 3 \\
1 / 2 \\
1 / 4 \\
0 \\
1 / 2 \\
1 / 8 \\
1 / 16 \\
1 / 8 \\
0 \\
1 / 2 \\
* \\
1 / 2 \\
1 / 4 \\
0 \\
1 / 2\end{array}$} & \multirow{3}{*}{$\begin{array}{c}2 / 7 \\
1 / 4 \\
3 / 8 \\
* \\
0 \\
1 / 6 \\
1 / 2 \\
0 \\
1 / 4 \\
5 / 16 \\
1 / 4 \\
1 / 2 \\
0 \\
3 / 8 \\
* \\
* \\
1 / 2 \\
0\end{array}$} & \multirow{3}{*}{$\begin{array}{c}2 / 7 \\
1 / 4 \\
1 / 4 \\
1 / 3 \\
1 / 2 \\
1 / 6 \\
0 \\
0 \\
3 / 8 \\
1 / 4 \\
1 / 4 \\
1 / 2 \\
0 \\
1 / 4 \\
1 / 2 \\
1 / 4 \\
0 \\
1 / 2\end{array}$} & \multirow{3}{*}{$\begin{array}{l}1 / 14 \\
1 / 4 \\
1 / 8 \\
1 / 6 \\
0 \\
1 / 4 \\
1 / 2 \\
1 / 2 \\
0 \\
1 / 8 \\
1 / 8 \\
0 \\
1 / 2 \\
1 / 8 \\
0 \\
1 / 4 \\
1 / 2 \\
0\end{array}$} \\
\hline $\begin{array}{l}12345 \\
1,2345 \\
2,1345 \\
3,1245 \\
123,45 \\
134,25 \\
235,14 \\
125,34 \\
1,23,45 \\
1,25,34 \\
2,13,45 \\
3,14,25\end{array}$ & $\begin{array}{c}0 \\
* \\
1 / 12 \\
1 / 8 \\
0 \\
0 \\
1 / 5 \\
0 \\
* \\
* \\
1 / 6 \\
1 / 8\end{array}$ & $\begin{array}{l}1 / 4 \\
1 / 4 \\
* \\
1 / 4 \\
1 / 4 \\
1 / 4 \\
1 / 10 \\
1 / 4 \\
1 / 4 \\
1 / 4 \\
* \\
1 / 4\end{array}$ & \begin{tabular}{|l|l}
$1 / 4$ \\
$1 / 4$ \\
$1 / 3$ \\
$*$ \\
$1 / 4$ \\
$1 / 4$ \\
$3 / 10$ \\
$1 / 4$ \\
$1 / 4$ \\
$1 / 4$ \\
$1 / 3$ \\
$*$
\end{tabular} & $\begin{array}{l}1 / 4 \\
1 / 4 \\
1 / 3 \\
3 / 8 \\
1 / 4 \\
1 / 4 \\
3 / 10 \\
1 / 4 \\
1 / 4 \\
1 / 4 \\
1 / 8 \\
3 / 8\end{array}$ & $\begin{array}{l}1 / 4 \\
1 / 4 \\
1 / 4 \\
1 / 4 \\
1 / 4 \\
1 / 4 \\
1 / 10 \\
1 / 4 \\
1 / 4 \\
1 / 4 \\
1 / 6 \\
1 / 4\end{array}$ & & & & & & \\
\hline \multicolumn{6}{|c|}{$\begin{array}{c}\text { Game No. } 4 \\
v(\{1,2,3\})=v(\{3,4,5\})=1\end{array}$} & & & & & & \\
\hline $\begin{array}{l}12345 \\
1,2345 \\
3,1245 \\
134,25\end{array}$ & $\begin{array}{c}1 / 6 \\
* \\
1 / 4 \\
0\end{array}$ & $\begin{array}{l}1 / 6 \\
1 / 3 \\
1 / 4 \\
1 / 3\end{array}$ & $\begin{array}{l}1 / 3 \\
1 / 3 \\
* \\
1 / 3\end{array}$ & $\begin{array}{l}1 / 6 \\
1 / 6 \\
1 / 4 \\
1 / 6\end{array}$ & $\begin{array}{l}1 / 6 \\
1 / 6 \\
1 / 4 \\
1 / 6\end{array}$ & \multicolumn{6}{|c|}{$\begin{array}{c}\text { Game No. } 8 \\
v(\{1,2,3\})=v(\{2,3,4\})=v(\{3,4,5\})= \\
v(\{1,4,5\})=v(\{1,2,5\})=1\end{array}$} \\
\hline $\begin{array}{l}125,34 \\
3,14,25 \\
1,34,25\end{array}$ & $\begin{array}{c}1 / 6 \\
1 / 6 \\
0 \\
1 / 2\end{array}$ & $\begin{array}{l}1 / 6 \\
1 / 6 \\
1 / 2 \\
0 \\
1 / 3\end{array}$ & $\begin{array}{r}1 / 3 \\
1 / 8 \\
* \\
* \\
1 / 3\end{array}$ & $\begin{array}{c}0 \\
1 / 6 \\
1 / 2 \\
0 \\
1 / 6\end{array}$ & $\begin{array}{l}1 / 9 \\
1 / 6 \\
0 \\
1 / 2 \\
1 / 6\end{array}$ & \multirow[t]{2}{*}{$\begin{array}{l}12345 \\
1,2345 \\
124,35 \\
1,24,35\end{array}$} & \multirow{2}{*}{$\begin{array}{c}1 / 5 \\
* \\
0 \\
1 / 6 \\
1 / 2 \\
* \\
*\end{array}$} & \multirow{2}{*}{$\begin{array}{c}1 / 5 \\
1 / 3 \\
1 / 2 \\
1 / 6 \\
0 \\
1 / 2 \\
1 / 4\end{array}$} & \multirow{2}{*}{$\begin{array}{c}1 / 5 \\
1 / 6 \\
0 \\
1 / 4 \\
1 / 2 \\
0 \\
1 / 4\end{array}$} & \multirow{2}{*}{$\begin{array}{c}1 / 5 \\
1 / 6 \\
0 \\
1 / 6 \\
0 \\
0 \\
1 / 4\end{array}$} & \multirow{2}{*}{$\begin{array}{c}1 / 5 \\
1 / 3 \\
1 / 2 \\
1 / 4 \\
0 \\
1 / 2 \\
1 / 4\end{array}$} \\
\hline$(\{2,3,4\})$ & $v(\{$ & & $=v$ & & $=1$ & & & & & & \\
\hline $\begin{array}{l}12345 \\
1,2345 \\
3,1245 \\
4,1235 \\
123,45 \\
135,24 \\
124,35\end{array}$ & $\begin{array}{c}1 / 9 \\
* \\
1 / 8 \\
1 / 6 \\
1 / 6 \\
1 / 12 \\
0 \\
1 / 6 \\
0 \\
1 / 12 \\
* \\
* \\
1 / 8 \\
0 \\
1 / 4\end{array}$ & $\begin{array}{l}1 / 9 \\
1 / 12 \\
1 / 4 \\
1 / 6 \\
1 / 12 \\
1 / 6 \\
1 / 6 \\
0 \\
1 / 6 \\
1 / 4 \\
1 / 6 \\
1 / 6 \\
1 / 4 \\
0 \\
1 / 4\end{array}$ & $\begin{array}{c}2 / 9 \\
1 / 4 \\
* \\
1 / 3 \\
1 / 4 \\
1 / 6 \\
1 / 6 \\
1 / 3 \\
1 / 6 \\
1 / 12 \\
1 / 6 \\
1 / 6 \\
* \\
1 / 2 \\
1 / 4\end{array}$ & $\begin{array}{c}1 / 3 \\
1 / 3 \\
3 / 8 \\
* \\
1 / 3 \\
1 / 3 \\
1 / 3 \\
1 / 3 \\
1 / 3 \\
1 / 3 \\
1 / 3 \\
1 / 3 \\
3 / 8 \\
* \\
*\end{array}$ & $\begin{array}{l}2 / 9 \\
1 / 3 \\
1 / 4 \\
1 / 3 \\
1 / 6 \\
1 / 4 \\
1 / 3 \\
1 / 6 \\
1 / 9 \\
1 / 4 \\
1 / 3 \\
1 / 3 \\
1 / 4 \\
1 / 2 \\
1 / 4\end{array}$ & & & & & & \\
\hline
\end{tabular}


TABLE 3

Pseudo-Kernels of Certain 3-Person Games

\begin{tabular}{c|c|c|c|c|c|c}
\multicolumn{8}{c}{$v(\{12\})=v(\{13\})=v(\{23\})=1$} \\
\cline { 1 - 4 }$v(\{123\})$ & $v(\{1\})$ & $v(\{2\})$ & $v(\{3\})$ & \multicolumn{3}{|c}{ Pseudo-Kernel } \\
\cline { 1 - 4 } $1 / 2$ & $1 / 2$ & $1 / 2$ & $1 / 2$ & $1 / 6$ & $1 / 6$ & $1 / 6$ \\
$1 / 2$ & $1 / 2$ & $1 / 2$ & 1 & $1 / 8$ & $1 / 8$ & $1 / 4$ \\
$1 / 2$ & $1 / 2$ & 1 & 1 & 0 & $1 / 4$ & $1 / 4$ \\
$1 / 2$ & 1 & 1 & 1 & $1 / 6$ & $1 / 6$ & $1 / 6$ \\
1 & $1 / 2$ & $1 / 2$ & $1 / 2$ & $1 / 3$ & $1 / 3$ & $1 / 3$ \\
1 & $1 / 2$ & $1 / 2$ & 1 & $1 / 4$ & $1 / 4$ & $1 / 2$ \\
1 & $1 / 2$ & 1 & 1, & 0 & $1 / 2$ & $1 / 2$ \\
1 & 1 & 1 & 1 & $1 / 3$ & $1 / 3$ & $1 / 3$ \\
\hline
\end{tabular}

the right column can be determined at a glance from the type font, without having to refer to the left column; the left column is retained chiefly to facilitate reference to the table.

The coalition structures that are listed for each game are of the types $\{a b c d e\}$ $\{a, b c d e\},\{a b c, d e\}$, and $\{a, b c, d e\}$. The kernels corresponding to some of the omitted ones can be obtained by applying the symmetries of the game to the tabulated kernels. In any other omitted structure, there is at most one non-flat coalition, and it has at most 3 players. Therefore by the Davis-Maschler "reduced game" technique explained in the previous section, the calculation of the corresponding kernel is equivalent to the calculation of the pseudo-kernel of a game with at most 3 players. If the game has exactly 3 players, then all 2-player coalitions have value 1. The pseudo-kernel may then be read off from Table 3, after applying the appropriate permutation. If the game has 2 players, then all 1-player coalitions have value 1 , and the pseudo-kernel is $(1 / 4,1 / 4)$ or $(0,0)$ according as the 2-player coalition has value $1 / 2$ or 0 .

The Hebrew University of Jerusalem

Weizmann Institute of Science,

Rehovoth, Israel

1. R. J. Aumann \& M. Maschler, "The bargaining set for cooperative games," Advance in Game Theory, M. Dresher, L. S. Shapley and A. W. Tucker, (Eds.), Annals of Mathematic Studies, No. 52, Princeton Univ. Press, Princeton, N. J., 1964, pp. 443-476.

2. M. I. BALINSKI, "An algorithm for finding all vertices of convex polyhedral sets," $J$. Soc. Indust. Appl. Math., v. 9, 1961, pp. 72-88. MR $25 * 5451$.

3. M. Davis \& M. Maschler, The Kernel of a Cooperative Game, Research Memorandum No. 58, Econometric Research Program, Princeton University, Princeton, N. J., June, 1963.

4. M. DAvis \& M. MASchler, "Existence of stable payoff configurations for cooperative games," "i2 Bull. Amer. Math. Soc., v. 69, 1963, pp. 106-108.

5. J. H. Griesmer, "Extreme games with three values," Contributions to the Theory of Games, Vol. IV, A. W. Tucker and R. D. Luce, (Eds.), Annals of Mathematics Studies, No. 40, Princeton Univ. Press, Princeton, N. J., 1959, pp. 189-212. MR $21 * 2539$.

6. H. M. GURK, "Five-person constant-sum extreme games," Contributions to the Theory of Games, Vol. IV, A. W. Tucker and R. D. Luce, (Eds.), Annals of Mathematics Studies, No. 40, Princeton University Press, Princeton, N. J., 1959, pp. 179-189. MR 21 * 1909.

7. J. R. ISBELL, "On the enumeration of majority games," $M T A C$, v. 13, 1959, pp. 21-28. MR $21 * 1912$.

8. R. D. LUCE \& H. RAIFFA, Games and Decisions; Introduction and Critical Survey, Wiley, New York, 1957. MR 19, 373.

${ }^{12} \mathrm{~A}$ detailed paper with the same title will appear in Studies in Mathematical Economics, Essays in Honor of Oskar Morgenstern, M. Shubik, editor. 
9. M. Maschler \& B. Peleg, "A characterization, existence proof, and dimension bounds for the kernel of a game," Pacific J. Math. (To appear.)

10. J. von Neumann \& O. Morgenstern, Theory of Games and Economic Behavior, Princeton Univ. Press, Princeton, N. J., 1944; 3rd ed., 1953. MR, 6 235; MR 9, 50.

11. B. Peleg, "Existence theorem for the bargaining set $\mathrm{M}_{1}(i), "{ }_{12}$ Bull. Amer. Math. Soc., v. 69, 1963, pp. 109-110. MR $26 * 2333$.

12. B. Peleg, "On the kernel of constant-sum games with homogeneous weights," Illinois J. Math. (To appear.)

13. B. Peneg, "The kernel of $m$-quota games," Canad. J. Math. Vol. 17, 1965, pp. 239-244.

14. L. S. ShaPLEY, "Simple games: an outline of the descriptive theory," Behavioral Sci., v. 7,1962 , pp. $59-66$. MR $24 *$ B2490. 